\title{
Factores de riesgo para el abandono del tratamiento de tuberculosis pulmonar sensible en un establecimiento de salud de atención primaria, Lima, Perú
}

\author{
Risk factors for drug-sensitive tuberculosis therapy \\ failure in a primary care health center in Lima, Peru
}

Correspondencia

Alexander Anduaga Beramend alexanderanduaga@hotmail.com

Recibido: 01/02/2016

Arbitrado por pares

Aprobado: 20/04/2016

\section{Citar como:}

Anduaga-BeramendiA, MaticorenaQuevedo J, Beas R, Chanamé-Baca $D M$, Veramendi $M$, Wiegering-

Rospigliosi A, Zevallos E, Cabrera $R$, Suárez-Ognio L. Factores de riesgo para el abandono del tratamiento de tuberculosis pulmonar sensible en un establecimiento de salud de atención primaria, Lima, Perú. Acta Med Peru. 2016;33(1):21-8
Alexander Anduaga-Beramendi ${ }^{1,2 a}$, Jesus Maticorena-Quevedo ${ }^{1,2, a}$, Renato Beas $^{1,2, a}$, Diego M. Chanamé-Baca ${ }^{1,2, a}$, Mary Veramendi ${ }^{1, a}$, Andrés WiegeringRospigliosi ${ }^{1, a}$, Erika Zevallos ${ }^{1, a}$, Rufino Cabrera ${ }^{1, b}$, Luis Suárez-Ognio ${ }^{1}$

1 Escuela de Medicina, Universidad Peruana de Ciencias Aplicadas. Lima, Perú.

2 Sociedad Científica de Estudiantes de Medicina de la Universidad Peruana de Ciencias Aplicadas (SOCIEMUPC). Lima, Perú

a Estudiante de medicina; b Biólogo

\section{RESUMEN}

Objetivo: Identificar los factores asociados al abandono del tratamiento antituberculoso en un centro de salud de atención primaria del distrito de Chorrillos, Lima, Perú. Materiales y métodos: Estudio de casos y controles retrospectivo no pareado. Se definió como caso a los pacientes que abandonaron el tratamiento por un periodo de 30 días consecutivos o más y como controles a los pacientes que terminaron el tratamiento antituberculoso. Se evaluó el puntaje de riesgo de abandono que se realiza en el centro de salud ( $\geq 22$ ), así como variables demográficas. Se calculó el odds ratio (OR) e intervalos de confianza al 95\% (IC95\%) usando regresión logística. Resultados: Se incluyó 34 controles y 102 casos. En el análisis multivariado se encontró que quienes cursaron menos de seis años de educación (OR: 22,2; IC95\%: 1,9-256,1) así como quienes tenían un puntaje $\geq a 22$ puntos en la prueba de riesgo de abandono $(O R=21,4 ; I C 95 \%: 6,3-72,4)$ tenían mayor probabilidad de abandonar el tratamiento antituberculoso. Conclusión: El abandono del tratamiento antituberculoso está asociado con tener menos de seis años de educación y un score mayor a 22 puntos en la prueba de abandono de tratamiento antituberculoso.

Palabras clave:

Tuberculosis; Factores de riesgo; Adhesión a la medicación; Tratamiento (fuente: DeCS BIREME). 


\begin{abstract}
Objective: To identify associated factors with failure of antituberculous therapy in a primary health center in Chorrillos, Lima, Peru. Material and methods: Retrospective case control study with a 1:3 case-control ratio. A case was defined as a patient who discontinued the treatment for a period of 30 consecutive days or more, and a control as a patient successfully completing antituberculous therapy. The score for risk of failure measured in the health center $(\geq 22)$ and demographic variables were assessed. Odds ratios (OR) and $95 \%$ confidence intervals $(\mathrm{Cl})$ were calculated using logistic regression. Results: Thirty-four cases and 102 controls were included. In the multivariate analysis, factors associated to a greater likelihood for a failure in antituberculous therapy were to have less than six years of education (OR: 22,2; $95 \%$ IC: $1,9-256,1)$ and to obtain a score greater or equal than 22 points in the test for risk of failure $(\mathrm{OR}=21,4 ; 95 \%$ IC: $6,3-72,4)$. Conclusions: Failure of antituberculous therapy was associated with having less than six years of education and a score greater than or equal to 22 points in the test for risk of failure.
\end{abstract}

Key words:

Tuberculosis; Riskfactors; Medication adherence; Therapy (source: MeSH NLM).

\section{INTRODUCCIÓN}

La tuberculosis (TB) es una infección crónica causada por la bacteria Mycobacterium Tuberculosis y desde el punto de vista clínico se han descrito las siguientes formas: TB pulmonar; TB extrapulmonar, que puede afectar cualquier órgano; la coinfección de TB con VIH y la TB subclínica [1]. La TB pulmonar es la forma más prevalente y su diseminación se realiza a través del aire cuando las personas infectadas expelen las bacterias [2]. Desde hace algunos años, han surgido cepas resistentes a los fármacos contra $M$. tuberculosis, constituyéndose cinco patrones de sensibilidad farmacológica: sensible a Isoniacida y Rifampicina; resistente a Isoniacida y Rifampicina o multidrogo resistente (MDR); resistente a Isoniacida, Rifampicina, cualquier fluoroquinolona y cualquier droga inyectable de segunda línea o extensivamente resistente (XDR); resistente a todas las drogas de primera y segunda línea o extremadamente resistente y la forma totalmente resistente [3].

Si bien la incidencia de TB ha caído en un promedio de $1,5 \%$ por año desde 2000, continúa, al lado del VIH, como una causa principal de mortalidad global [2,4]. En América del Sur, el mayor número de casos y las tasas más elevadas de incidencia de esta enfermedad se concentran en Brasil y Perú [4,5]. Para el año 2014, en el Perú se reportaron 30008 nuevos casos según el reporte mundial de TB [2]. Respecto de las formas resistentes a fármacos, se estima que $3,3 \%$ de los nuevos casos diagnosticados de TB a nivel mundial son TB MDR, de los cuales $9,7 \%$ son de la forma XDR [2]. En nuestro país, en los últimos años se han reportado 1500 casos de TB MDR y 80 casos de TB XDR por año; siendo la mayoría de estos notificados por Lima y Callao $[1,6]$.

Con respecto al manejo de la TB en el Perú, como protocolo, se realiza la baciloscopia de esputo y una prueba rápida de sensibilidad molecular o MODS (Microscopic Observation Drug Susceptibility), previas a la asignación del tratamiento respectivo [7]. El tratamiento para TB sensible, de acuerdo al Ministerio de Salud del Perú (MINSA), consiste en Isoniacida (H), Rifampicina (R), Pirazinamida (Z) y Etambutol (E) , y consta de dos fases: la primera de manera diaria con HREZ por dos meses; y la segunda fase tres veces por semana con HR, durante cuatro meses [7]. Este tratamiento brindado por el MINSA es de forma gratuita y se entrega bajo supervisión del personal de salud [7].

Una de las principales barreras para el éxito del tratamiento antes mencionado ha sido la tasa de abandono al tratamiento que para el año 2012 alcanzaba el 5\%, siendo incluso mayor en algunos departamentos del Perú [8]. Entre los determinantes del abandono al tratamiento se ha descrito que el tiempo de duración de éste, los efectos adversos de las drogas, los pacientes con peores condiciones de vida, la pobreza, el desempleo y la mayor edad conllevan a menor probabilidad de continuar el tratamiento [911]. Otras causas son la insuficiente información proporcionada al paciente por el personal de salud sobre el tratamiento, los horarios inadecuados para la administración del mismo, el consumo de drogas, la secundaria incompleta, el sexo masculino y tener el antecedente de abandono previo $[7,8,10,12]$.

A pesar de que se han publicado algunos estudios sobre los factores de riesgo de abandono de tratamiento de la TB pulmonar sensible en el Perú, aún es necesario realizar más indagaciones con el fin de establecer estrategias para reducir el abandono del tratamiento $y$, en consecuencia, la resistencia a los antituberculosos. El objetivo del presente estudio fue identificar los factores de riesgo para abandono del tratamiento de TB pulmonar sensible en un centro de salud de atención primaria del departamento de Lima, Perú.

\section{MATERIALES Y MÉTODOS}

\section{Diseño del estudio}

Estudio observacional, diseño casos y controles no pareado con una razón caso-control de 1 a 3 . El estudio se basó en fuente secundaria (historias clínicas) de pacientes con TB pulmonar que iniciaron tratamiento antituberculoso (Esquema I) entre los años 2004 y 2013 en el Centro de Salud San Genaro de Villa, Chorrillos, 


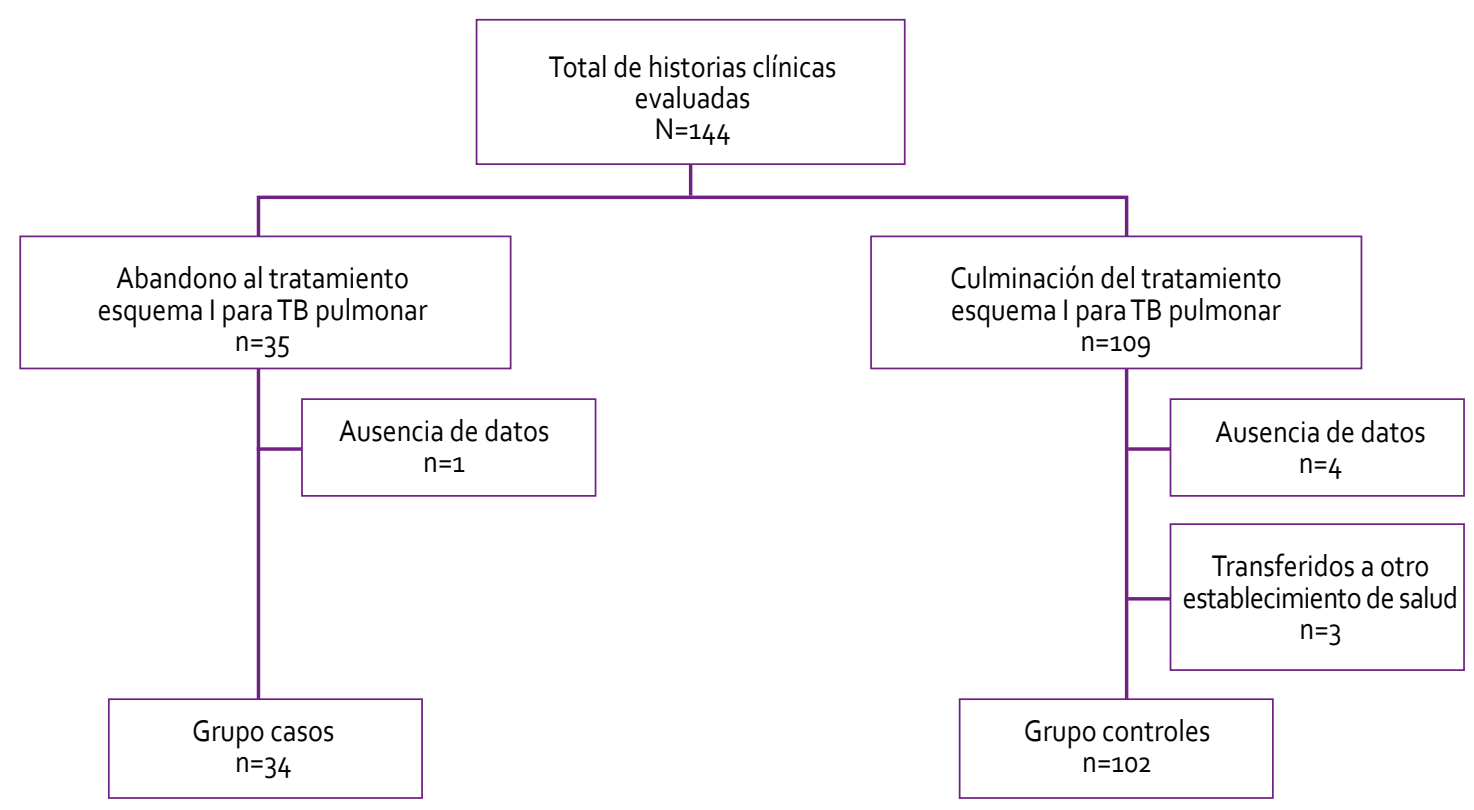

Figura 1. Flujograma de casos y controles.

Lima - Perú. La identificación y revisión de las historias clínicas se realizó desde Septiembre hasta Noviembre de 2014.

\section{Población y tamaño de muestra}

Se incluyeron en el estudio los pacientes mayores de 18 años con diagnóstico de TB pulmonar, que iniciaron tratamiento directamente observado (DOTS) entre los años 2004 y 2013 en el Centro de Salud San Genaro de Villa, Chorrillos con el Esquema I - isoniacida $(H)$, rifampicina $(R)$, pirazinamida (Z) y etambutol (E). Asimismo, se excluyeron los pacientes que cumplían con los siguientes criterios: fallecidos durante el tratamiento antituberculoso, TB extrapulmonar, TB en cualquiera de sus formas resistentes, referidos o contrareferidos de otros centros de salud, y aquellos con historias incompletas.

El tamaño de la muestra se calculó con el software openepi.com versión 3.01 para un estudio de casos y controles no pareado, utilizando los siguientes criterios: intervalo de confianza de 95\%, poder estadístico de $80 \%$, una relación caso: control de 1:2, la proporción de exposición en los controles de 3,39\%, la proporción de exposición en los casos de 25,86\% y un OR de 9,94 [9]. El tamaño inicial de la muestra fue de 96 personas ( 32 casos y 64 controles), sin embargo, debido que el número de casos era menor de lo esperado, se estableció una relación caso control de 1 a 3 obteniéndose así, 34 casos y 102 controles.

Los pacientes que abandonaron el tratamiento por un periodo de 30 días consecutivos o más, fueron categorizados como casos, y los pacientes que terminaron el tratamiento antituberculoso satisfactoriamente fueron categorizados como controles; ambos documentados en las historias clínicas [12]. Tanto los casos como los controles se seleccionaron del mismo año mediante los criterios de inclusión y por conveniencia de los investigadores. Figura 1.

\section{Definiciones operacionales de las variables principales}

La población incluida recibió tratamiento para tuberculosis pulmonar sensible con el Esquema I de acuerdo a la norma técnica del MINSA del año 2013, el cual incluye: isoniacida $(H)$, rifampicina $(R)$, pirazinamida $(Z)$ y etambutol $(E)$ por 6 meses dividido en dos etapas [7]. La primera fase de dos meses (diario de lunes a sábado con HRZE) y la segunda de cuatro meses (tres veces por semana con $\mathrm{RH}$ ) [7].

Se consideró el riesgo de abandono según los valores mostrados en la Tabla 1. Esta variable fue tomada a partir de la evaluación psicológica de la Estrategia Sanitaria Nacional de Prevención y Control de la Tuberculosis (PCT), de la Red de salud Barranco, Chorrillos, Surco de la Dirección de salud (DISA) II Lima Sur del Ministerio de Salud del Perú [13]. El instrumento evalúa psicológicamente al paciente mediante un conjunto de cuestionarios:

- ElTest Rápido de Funciones Mentales MAJOI: evalúa las áreas de memoria, afecto, juicio, orientación e intelecto del paciente.

- El Test Rápido de Depresión: se asemeja al cuestionario de Yesavage abreviado [14]. Contiene 15 ítems y es usado para despistaje de depresión.

- Conductas de riesgo: evalúa la frecuencia del consumo de alcohol, drogas y tabaco

- Conducta sexual de riesgo: evalúa el número de parejas sexuales, uso de métodos de protección y relaciones sexuales con personas del mismo sexo.

- Conciencia de enfermedad: mediante la clasificación McEvoy [15]. Evalúa el conocimiento de la enfermedad y los síntomas, así como su aceptación. 
Tabla 1. Escala de riesgo de abandono de la evaluación psicológica del PCT de la DISA II Lima, Perú.

\begin{tabular}{ll}
\multicolumn{1}{c}{ Riesgo de abandono } & \multicolumn{1}{c}{ Puntaje } \\
\hline Bajo riesgo de abandono & $<22$ puntos \\
Mediano riesgo abandono & De 22 a 36 puntos \\
Alto riesgo de abandono & $>36$ puntos \\
\hline
\end{tabular}

- Grado de adherencia: Evalúa la toma correcta de la medicación y si es de manera autónoma o necesita supervisión para la toma adecuada

- Dispone de informes de evaluación: Corrobora si el paciente ha sido evaluado por todo el equipo multidisciplinario del PCT o no.

- Situación al alta: Dividido en dos secciones. Una evalúa la evolución clínica y psicológica del paciente y la otra evalúa si el paciente se encuentra hospitalizado o es tratado ambulatoriamente.

Cada evaluación tiene un puntaje determinado el cual se suma para determinar el riesgo de abandono del tratamiento de TB.

El antecedente de abandono del tratamiento previo fue definido como el haber abandonado anteriormente un tratamiento establecido con fármacos anti-tuberculosos consignado en la historia clínica. El antecedente de infección por VIH fue definido como el resultado de ELISA reactivo para VIH y confirmado por Western Blot verificado en la historia clínica. Asimismo, el alcoholismo, el uso de drogas y el hábito de consumir tabaco fueron recogidas de la historia clínica.

\section{Análisis de los datos}

Las variables recogidas en la ficha de investigación fueron ingresadas en una base de datos elaborada en el programa Microsoft Excel $2010^{\circledR}$. El control de calidad se realizó mediante la doble tabulación y confrontación posterior de los datos. Los datos obtenidos se almacenaron y procesaron en el paquete estadístico STATA $13^{\circledR}$.

Las variables riesgo de abandono e ingresos mensuales se dicotomizaron, la edad se categorizó en terciles y el nivel educativo se categorizó en tres categorías de acuerdo a la cantidad de años estudiados (< de 6,6 a 11 y > de 11). La variable riesgo de abandono se dicotomizó en riesgo alto, y riesgo moderado más riesgo bajo; ya que todos los participantes que tenían riesgo alto habían abandonado el tratamiento antituberculoso. Para el análisis bivariado se empleó la prueba estadística de Chi2 y la prueba exacta de Fisher de acuerdo a los valores esperados. Asimismo, se calcularon los odds ratio (OR) con un IC 95\%. Adicionalmente, se realizó un análisis multivariado mediante regresión logística ajustando las variables que se encontraron con un $p<0,20$ en el análisis bivariado. El ajuste del modelo se comprobó con el test de Hosmer-Lemenshow.

\section{Aspectos éticos}

El estudio fue aprobado por el Comité de Ética de la Universidad Peruana de Ciencias Aplicadas (UPC): Pl165-2014. Para el recojo de información y el desarrollo de la investigación se contó con la autorización del Centro de Salud San Genaro de Villa. Asimismo, en el registro se omitió cualquier dato que permita la identificación de los pacientes. Debido a que la investigación se basó en la revisión de historias clínicas, no se aplicó consentimiento informado.

\section{RESULTADOS}

Se identificaron 136 historias clínicas (34 casos y 102 controles) entre el año 2004 y 2013 . Del total de participantes, 85 (62,5\%) fueron de sexo masculino. La media de la edad fue 29,8 con valor mínimo de 18 y máximo de 80 años, con una desviación estándar de 12,9. La media de la edad para los casos fue 30,9 y para controles 29,4 años. De los participantes, $78(57,4 \%)$ eran solteros/as y $100(73,5 \%)$ habían completado la educación secundaria. En cuanto a la ocupación, $30(22,1 \%)$ eran ama/o de casa y $21(15,4 \%)$ estudiantes. Por otro lado, se encontró un $3,7 \%$ con antecedentes de abandono al tratamiento antituberculoso, $27,2 \%$ presentaba alguna comorbilidad y $5,2 \%$ era positivo para $\mathrm{VIH}$. Con respecto a los hábitos nocivos, se encontró que el 29,4\% tenía historia de alcoholismo, el 11,8\% consumía drogas y un $19,1 \%$ tenía historia de tabaquismo.

Las variables que fueron significativas al realizar el análisis bivariado fueron: años de estudio, ingreso económico mensual y riesgo de abandono. En el análisis crudo se encontró que años de estudio de $<6$ (OR= 23,22 IC: 3,98-135,55), tener ingresos mensuales menores de 700 soles (OR=3,74 IC: $1,34-10,47)$, y obtener un puntaje $\geq$ a 22 puntos en la prueba de riesgo de abandono $(\mathrm{OR}=21,9, \mathrm{IC} 95 \% 7,05-68,1)$ estuvieron asociados al abandono del tratamiento. Tabla 2.

En el análisis multivariado se mantuvo la asociación entre riesgo de abandono según el test de riesgo para la DISA II del MINSA, y años de estudio $<6$, pero se perdió para ingresos mensuales menores a 700 soles. La bondad del ajuste propuesto a través del test de Hosmer-Lemeshow explica que lo observado es correcto. Tabla 3.

\section{DISCUSIÓN}

El manejo de la TB como endemia en el país obedece, en gran parte, al cumplimiento y éxito del tratamiento. Naturalmente, el abandono al tratamiento de la TB se relaciona con el mal pronóstico del paciente. En el presente estudio realizado en un centro de atención primaria de Lima, se han identificado factores de riesgo relacionados al ambiente como nivel educativo y al paciente como el nivel de riesgo de abandono según la evaluación psicológica mencionada anteriormente, que influyen en la decisión de abandonar o continuar el tratamiento. 
Tabla 2. Factores asociados al abandono del tratamiento de la TB pulmonar sensible en el Centro de Salud de Chorrillos, Lima, Perú 2004-2013.

\begin{tabular}{|c|c|c|c|c|c|c|c|}
\hline \multirow[t]{2}{*}{ Variables } & \multicolumn{2}{|c|}{ Casos } & \multicolumn{2}{|c|}{ Controles } & \multirow[t]{2}{*}{$p$} & \multirow[t]{2}{*}{ OR } & \multirow[t]{2}{*}{ (IC 95\%) } \\
\hline & $n$ & $(\%)$ & $\bar{n}$ & $(\%)$ & & & \\
\hline Edad & & & & & 0,571 & & \\
\hline $18-22$ & 9 & $(26,5)$ & 37 & $(36,3)$ & & Ref & \\
\hline $23-29$ & 13 & $(38,2)$ & 35 & $(34,3)$ & & 1,53 & $(0,58-4,01)$ \\
\hline $30-80$ & 12 & $(35,3)$ & 30 & $(29,4)$ & & 1,64 & $(0,61-4,42)$ \\
\hline Sexo & & & & & 0,759 & & \\
\hline Masculino & 22 & $(64,7)$ & 63 & $(61,8)$ & & Ref & \\
\hline Femenino & 12 & $(35,3)$ & 39 & $(38,2)$ & & 0,88 & $(0,39-1,98)$ \\
\hline Co-morbilidad & & & & & 0,243 & & \\
\hline $\mathrm{Si}$ & 13 & $(38,2)$ & 24 & $(23,5)$ & & Ref & \\
\hline No & 10 & $(29,4)$ & 35 & $(34,3)$ & & 0,53 & $(0,20-1,40)$ \\
\hline Ignorado & 11 & $(32,3)$ & 43 & $(42,2)$ & & 0,47 & $(0,18-1,22)$ \\
\hline Años de estudio* & & & & & $<0,001$ & & \\
\hline$<6$ años & 11 & $(32,3)$ & 3 & $(2,94)$ & & 23,22 & $(3,98-135,55)$ \\
\hline 6-11 años & 20 & $(58,8)$ & 80 & $(78,4)$ & & $1,5^{8}$ & $(0,43-5,88)$ \\
\hline > 11 años & 3 & $(8,8)$ & 19 & $(18,6)$ & & Ref & \\
\hline Estado civil* & & & & & 0,660 & & \\
\hline Soltero & 20 & $(58,8)$ & 58 & $(56,9)$ & & Ref & \\
\hline Casado & 7 & $(20,6)$ & 19 & $(18,6)$ & & 1,07 & $(0,40-2,91)$ \\
\hline Viudo & 2 & $(5,9)$ & 3 & $(2,90)$ & & 1,93 & $(0,30-12,42)$ \\
\hline Divorciado & 0 & (0) & 1 & $(1,0)$ & & - & - \\
\hline Separado & 1 & $(2,9)$ & 1 & $(1,0)$ & & 2,90 & $(0,17-48,56)$ \\
\hline Conviviente & 4 & $(11,8)$ & 20 & $(19,6)$ & & 0,58 & $(0,18-1,90)$ \\
\hline Ocupación* & & & & & 0,463 & & \\
\hline Obrero & 5 & $(14,7)$ & 11 & $(10,8)$ & & Ref & \\
\hline Estudiante & 3 & $(8,8)$ & 18 & $(17,6)$ & & 0,37 & $(0,07-1,84)$ \\
\hline Profesor & 1 & $(0,98)$ & 1 & $(2,94)$ & & 2,2 & $(0,11-42,70)$ \\
\hline Ama de casa & 6 & $(17,6)$ & 24 & $(23,5)$ & & 0,55 & $(0,14-2,19)$ \\
\hline Otros & 19 & $(55,9)$ & 48 & $(47,1)$ & & 0,87 & $(0,27-2,84)$ \\
\hline Ingresos mensuales & & & & & 0,009 & & \\
\hline 700 a más & 5 & $(14,7)$ & 40 & $(39,2)$ & & Ref & \\
\hline Menor a 700 & 29 & $(85,3)$ & 62 & $(60,8)$ & & 3,74 & $(1,34-10,47)$ \\
\hline Antecedente de abandono de tratamiento* & & & & & 0,99 & & \\
\hline Si & 1 & $(2,9)$ & 4 & $(3,9)$ & & Ref & \\
\hline No & 33 & $(97,1)$ & 98 & $(96,1)$ & & 0,74 & $(0,08-6,88)$ \\
\hline Infección por VIH* & & & & & 0,065 & & \\
\hline $\mathrm{Si}$ & 4 & $(11,8)$ & 3 & $(2,9)$ & & 4,40 & $(0,93-20,77)$ \\
\hline No & 30 & $(88,2)$ & 99 & $(97,1)$ & & Ref & \\
\hline Alcoholismo & & & & & 0,99 & & \\
\hline Si & 10 & $(39,4)$ & 30 & $(39,4)$ & & 1 & $(0,43-2,34)$ \\
\hline No & 24 & $(70,6)$ & 72 & $(70,6)$ & & Ref & \\
\hline Consumo de drogas* & & & & & 0,229 & & \\
\hline Si & 6 & $(17,7)$ & 10 & $(9,8)$ & & 1,97 & $(0,66-5,90)$ \\
\hline No & 28 & $(82,4)$ & 92 & $(90,2)$ & & Ref & \\
\hline Consumo de tabaco & & & & & 0,801 & & \\
\hline Si & 6 & $(17,7)$ & 20 & $(19,6)$ & & 0,88 & $(0,32-2,41)$ \\
\hline No & 28 & $(82,4)$ & 82 & $(80,4)$ & & Ref & \\
\hline Riesgo de abandono & & & & & $<0,001$ & & \\
\hline Moderado+Alto $(\geq 22)$ & 30 & $(88,2)$ & 26 & $(25,5)$ & & 21,92 & $(7,05-68,16)$ \\
\hline Bajo (<22) & 4 & $(11,8)$ & 76 & $(74,5)$ & & Ref & \\
\hline
\end{tabular}


Tabla 3. Análisis multivariado TB pulmonar sensible en el Centro de Salud de Chorrillos, Lima, Perú 2004-2013.

\begin{tabular}{lrcc}
$\begin{array}{lrc}\text { Abandono al tratamiento anti-TB } \\
\text { Ingresos }<700\end{array}$ & OR & (IC 95\%) & p \\
$\begin{array}{l}\text { Nivel de educación } \\
\quad \text { 11 años }\end{array}$ & Ref & & 0,956 \\
$\quad 6-11$ años & 0,96 & $(0,29-3,75)$ & \\
$\quad<6$ años & 22,19 & $(1,92-256,10)$ & 0,013 \\
VIH & 1,18 & $(0,12-11,19)$ & 0,883 \\
Riesgo de abandono & 21,36 & $(6,30-72,42)$ & $<0,001$ \\
\hline
\end{tabular}

En el análisis ajustado se encontró asociación entre el abandono de tratamiento de la TB y los años de estudio menor de 6 años (OR: 22,19). Se conoce por estudios realizados en Nicaragua y Brasil, que el abandono de tratamiento de la TB se encuentra relacionado al antecedente de tener menor grado de educación [16-18]. Esta asociación puede deberse al menor conocimiento de la enfermedad y menor acceso a información que podrían tener estos pacientes, lo cual conlleva a baja adherencia al tratamiento por parte de esta población [19,20]. Asimismo, gran parte de los pacientes con menor nivel educativo poseen menores ingresos económicos. En nuestra población, si bien 13 $(92,9 \%)$ de quienes tenían menos de 6 años de estudio percibían ingresos menores a 700 soles, los ingresos mensuales no mostraron asociación en el análisis multivariado. En la literatura se menciona que la dificultad al acceso a servicios de salud por posibles pérdidas en días de trabajo y los gastos de transporte son los principales motivos por los que las personas con bajos ingresos mensuales no acuden a recibir tratamiento $[20,21]$.

En el análisis del consumo de sustancias psicoactivas no se encontró asociación entre su uso y el riesgo de abandono al tratamiento. Contrariamente, diversos estudios indican que el consumo de dichas sustancias podría estar asociado al abandono del tratamiento, debido al comportamiento de riesgo y modo de vida que presenta este grupo de personas [20,22,23]. Asimismo, el alcoholismo, el cual es un factor predisponente del desarrollo de la TB y está asociado a falla al tratamiento y peor pronóstico, no se mostró asociado a un mayor riesgo de abandono [24-26].

Otro factor de riesgo asociado fue tener un puntaje $\geq 22$ en la evaluación psicológica del PCT del MINSA $(O R=21,36)$. Se considera importante esta herramienta debido a que no sólo permite identificar pacientes con alto riesgo de abandono, sino valora también de forma independiente diversos aspectos. La depresión, que es uno de estos aspectos, se ha reportado asociado a una menor adherencia al tratamiento antituberculoso en policlínicos de la Red Almenara EsSalud de Lima, además se relaciona a tasas considerables de ideación suicida $(9,0 \%)$ e historia de intento de suicidio $(3,1 \%)$ en pacientes con tuberculosis que reciben tratamiento en centros de atención primaria [27-29]. Asimismo, un estudio realizado por Silva et al en un estado de Brasil reportó asociación entre ser portador de una enfermedad mental y el abandono del tratamiento de la TB, lo cual refuerza la importancia de esta evaluación previa al inicio del tratamiento [30]. Otro aspecto evaluado dentro de este instrumento es la conciencia de la enfermedad, que es un aspecto básico asociado previamente a la falla al tratamiento antituberculoso y está relacionado, también, a la deficiente educación brindada por el personal de salud a los pacientes [31]. En ese sentido, la evaluación psicológica integral del paciente en tratamiento antituberculoso es muy relevante, un estudio demostró que más de la mitad de estos pacientes presentó grados de malestar psicológico alto o mayor $[32,33]$.

En la bibliografía también se resalta asociación entre la coinfección VIH y TB, sin embargo este dato relacionado a la biología humana no se confirmó en el análisis [34]. Lo anterior podría ser consecuencia de la baja prevalencia de pacientes VIH positivos de la muestra, y la mayor prevalencia de VIH en pacientes con TB-MDR Y TB-XDR, quienes fueron excluidos. De la misma forma, las variables ocupación, edad, estado civil y sexo, que también se midieron en el estudio no presentaron asociación.

Los resultados del presente estudio deben evaluarse teniendo en cuenta ciertas limitaciones: la recolección de datos se llevó a cabo a partir de historias clínicas que podrían haber sido llenadas inadecuadamente por personal de salud; asimismo, el tamaño muestral y la circunscripción a un determinado distrito de Lima podría limitar la extrapolación de resultados. Por otro lado, no fue posible evaluar cada componente de la evaluación psicológica del PCT del MINSA de manera independiente por la carencia de los puntajes específicos en cada categoría; lo cual debería considerarse para posteriores investigaciones con la intención de revisar y validar el instrumento. Sin embargo, el análisis de la asociación entre el nivel de riesgo de abandono según esta evaluación y el abandono del tratamiento constituye un aspecto no evaluado anteriormente. Asimismo, el intervalo de tiempo considerado para el estudio permite que los resultados reflejen la situación de los últimos años.

En conclusión, el estudio muestra que el paciente con mayor probabilidad de abandonar el tratamiento de la TB pulmonar 
sensible, pertenece al grupo poblacional de personas con menos de 6 años de estudios. Asimismo, se revela la asociación entre haber obtenido un puntaje $\geq 22$ en la evaluación psicológica de riesgo de abandono establecida por la DISA II Lima Sur del MINSA y el abandono del tratamiento de la TB. Este estudio ofrece nuevas posibilidades de investigación relacionadas a la utilización de la Evaluación Psicológica del PCT con el propósito de validar su importancia para predecir e intervenir oportunamente a pacientes con alto riesgo de abandonar el tratamiento. Lo anterior, permitirá la aplicación más amplia, y no solo por parte de la Red Barranco - Chorrillos - Surco de la DISA II Lima Sur, del instrumento mencionado para mejorar el seguimiento de los pacientes, así como disminuir la tasa de abandono.

\section{AGRADECIMIENTOS}

A la doctora Elsa Ibarra Casablanca, coordinadora del curso de Médico Paciente 6, por las facilidades brindadas para el desarrollo del trabajo campo. Al psicólogo Ricardo Mejía Saona por la explicación en la realización y funcionamiento del test de riesgo de abandono. A la psicóloga Martha Guevara por darnos las facilidades para el acceso al centro de salud San Genaro de Villa. Al centro de salud San Genaro de Villa por brindarnos las facilidades para nuestra investigación. A nuestra compañera Ana Lucía Ayasta Monge por su colaboración en la recolección de datos para esta investigación.

Conflictos de interés: Los autores declaran que no hay ningún conflicto de interés en la publicación de este artículo.

Fuente de financiamiento: Autofinanciado

\section{REFERENCIAS BIBLIOGRÁFICAS}

1. Zumla A, Raviglione $M$, Hafner R, von Reyn CF.. Tuberculosis. N Engl J Med. 2013;368(8):745-55.

2. World Health Organization (WHO). Global Tuberculosis Report 2015. Geneva: World Health Organization; 2015.

3. Dheda K, GumboT, Gandhi NR, Murray M, Theron G, Udwadia Z, et al. Global control of tuberculosis: from extensively drug-resistant to untreatable tuberculosis. Lancet Respir Med. 2014i2(4):321-38.

4. Dye C. Global burden on TB: estimated incidence, prevalence and mortality by country. JAMA. 1999;282(7):677-86.

5. Suárez P, Watt C, Alarcón E, Portocarrero J, Zavala D, Canales R et al. The dynamics of TB in response to 10 years of intensive control effort in Peru. J Infect Dis. 2001;184(4):473-8.

6. Perú, Ministerio de Salud. Situación de TB en el Perú [Internet]. Lima: MINSA; 2011 [citado el 12 de enero de 2016]. Disponible en: http://www.minsa.gob.pe/portada/Especiales/2011/respiravida/ archivos/Ayuda_memoria_Lanzamiento_TB.pdf

7. Perú, Ministerio de Salud Norma Técnica de Salud para la atención integral de las personas afectadas por TB. Resolución Ministerial $N^{\circ}$ 715-2013/MINSA del 8 de noviembre del 2013. Lima: MINSA; 2013.

8. Culqui DR, Munayco CV, Grijalva CG, et al. Factors associated with the non-completion of conventional anti-TB treatment in Peru. Arch Bronconeumol. 2012;48(5):150-5.
9. Culqui DR, Grijalva CG, Reátegui SR, Cajo JM, Suárez LA. Factores pronósticos del abandono del tratamiento antituberculoso en una región endémica del Perú. Rev Panam Salud Publica. 2005;18(1):1420.

10. Bonilla C. Situación de la TB en el Perú. Acta Med Peruana. 2008;25(3):163-70.

11. Perú, Ministerio de Salud. Programa Nacional de Control de Enfermedades Transmisibles - Control de la TB. Actualización de la doctrina, normas y procedimientos para el control de la TB en el Perú. Lima: MINSA; 2001.

12. Muture BN1, Keraka MN, Kimuu PK, Kabiru EW, Ombeka VO, et al. Factors associated with default from treatment among TB patients in Nairobi province, Kenya: a case control study. BMC Public Health. 2011;11:696.

13. Dirección General de Salud de las Personas, Ministerio de Salud. Reunión de trabajo: Participación del psicólogo en el Comité de Evaluación y Retratamiento Intermedio (CERI) en Lima y Callao [internet]. Lima: MINSA; 2009 [citado el 26 de noviembre de 2014]. Disponible en: ftp://ftp2.minsa.gob.pe/descargas/dgsp/ ESN-tuberculosis/sm/PsicologoCERI.pdf

14. J. Martínez De La Iglesia, C. Onís Vilches, R. Dueñas Herrero, C. Albert Colomer, C. Aguado Taberné, R. Luque Luque. Versión española del cuestionario de Yesavage abreviado (GDS) para el despistaje de depresión en mayores de 65 años: adaptación y validación. MEDIFAM. 2002;12(10):620-30.

15. McEvoy JP. Organic brain syndromes. Ann Intern Med. 1981;95(2):212-20.

16. Jimenez GD, López PD, Medrano MJ, Valle MJ. Factores asociados al abandono de pacientes bacillíferos al programa de control de tuberculosis, Managua, Nicaragua, 1998-1999. Managua: Centro de Investigaciones y Estudios de la Salud (CIES-UNAN); 1992.

17. Steffen R, Menzies D, Oxlade O, Pinto M, de Castro AZ, Monteiro $P$, et al. Patients' costs and cost-effectiveness of tuberculosis treatment in DOTS and non-DOTS facilities in Rio de Janeiro, Brazil. PLoS One. 2010;5(11):e14014.

18. Orofino Rde L, Brasil PE, Trajman A, Schmaltz CA, Dalcolmo $\mathrm{M}$, et al. Predictors of tuberculosis treatment outcomes. J Bras Pneumol. 2012;38(1):88-97.

19. Arriola-Huerta P, Castillo-Cahuana T, Quispe-Fabián G, Torres-Deza C. Factores asociados a la asistencia del paciente al tratamiento antituberculoso. Rev Enferm Herediana. 2011;4(2):86-92.

20. Albuquerque $M$, Ximenes $R$, Lucena-Silva $N$, Souza $W$, Dantas Andréa $T$, et al. Factors associated with treatment failure, dropout, and death in a cohort of tuberculosis patients in Recife, Pernambuco State, Brazil. Cad Saude Publica. 2007;23(7):1573-82.

21. Caylà JA, Caminero JA, Rey $R$, Lara N, Vallés $X$, et al. Current status of treatment completion and fatality among tuberculosis patients in Spain. Int J Tuberc Lung Dis. 2004;8(4):458-64.

22. Bam TS, Gunneberg C, Chamaroonsawasdi K, Bam DS, Aalberg $\mathrm{O}$, et al. Factors affecting patient adherence to DOTS in urban Kathmandu Nepal. Int J Tuberc Lung Dis. 2006;10(3):270-6.

23. Godoy $P$, Nogues A, Alseda M, Manonelles A, Artigues A, et al. Factores de riesgo asociados a pacientes tuberculosos con microscopia de esputo positiva. Gac Sanit. 2001;15(6):506-12.

24. Przybylski G, Dąbrowska A, Trzcińska H. Alcoholism and other socio-demographic risk factors for adverse TB-drug reactions and unsuccess full tuberculosis treatment - data from ten years' observation at the Regional Centre of Pulmonology, Bydgoszcz, Poland. Med Sci Monit. 2014;20:444-53.

25. Daniel OJ, Oladapo OT, Alausa OK. Default from treatment programme in Sagamu, Nigeria. Niger J Med. 2006;15(1):63-7. 
26. Slama K, Tachfouti N, Obtel M, Nejjari C. Factors associated with treatment default by tuberculosis patients in Fez, Morocco. East Mediterr Health J. 2013;19(8):687-93.

27. Peltzer K, Louw J. Prevalence of suicidal behaviour \& associated factors among tuberculosis patients in public primary care in South Africa. Indian J Med Res. 2013;138(2):194-200.

28. Rajeswari R, Muniyandi M, Balasubramanian R, Narayanan PR. Perceptions of tuberculosis patients about their physical, mental and social well-being: a field report from south India. Soc Sci Med 2005;60(8):1845-53.

29. Morales H. Influencia de la depresión sobre la adherencia al tratamiento en pacientes TB-MDR de la Red Almenara. Revista de la Facultad de Medicina Humana de la Universidad Ricardo Palma. 2015;1:16-22.

30. Silva PdF, Moura GS, Caldas AdJM. Fatores associados ao abandono do tratamento da tuberculose pulmonar no Maranhão, Brasil, no período de 2001 a 2010. Cad Saude Publica. 2014;30(8):1745-54.
31. Morsy AM, Zaher HH, Hassan MH, Shouman A. Predictors of treatment failure among tuberculosis patients under DOTS strategy in Egypt. East Mediterr Health J. 2003;9(4):689-701.

32. Aguedo A, Nattaly S. Grado de malestar psicológico en pacientes con tuberculosis de tres centros de salud de Lima, 2014. Lima: Facultad de Medicina, Universidad Nacional Mayor de San Marcos; 2015.

33. Perú, Ministerio de Salud. La Salud Mental Como Predictor De Adherencia Al Tratamiento Antituberculosis. 2012 [Internet]. Lima: MINSA; C2014 [citado el 26 de noviembre de 2014]. Disponible en: $\mathrm{ftp}: / / f t p 2 . m i n s a . g o b . p e / n o r m a s l e g a l e s / 2010 / R M 579-2010-M I N S A$. pdf

34. Fry RS, Khoshnood K, Vdovichenko E, Granskaya J, Sazhin V Shpakovskaya L. Barriers to completion of tuberculosis treatment among prisoners in St. Petersburg, Rusia. Int J Tuberc Lung Dis. 2005;9(9):1027-33.

\section{Las ediciones anteriores de Acta Médica Peruana están disponibles en:}

\section{www.scielo.org.pe}

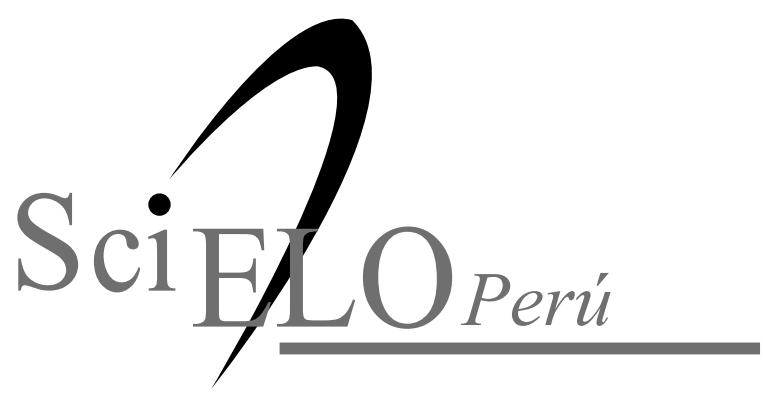

